Article

\title{
Why Naked Mole-Rats Have High Oxidative Damage but Live a Long Life: A Simple Explanation Based on the Oxidative Stress Theory of Aging
}

\author{
Wenyun Zuo ${ }^{1}$, Xiaolong Tang ${ }^{2}$, Chen Hou ${ }^{3, *}$ \\ 1 Department of Biology, Stanford University, Stanford, CA 94305, USA \\ 2 Institute of Biochemistry and Molecular Biology, School of Life Sciences, \\ Lanzhou University, Lanzhou, 730000, China \\ 3 Department of Biological Sciences, Missouri University of Science and \\ Technology, Rolla, MO 65409, USA \\ * Correspondence: Chen Hou, Email: houch@mst.edu.
}

\section{G Open Access}

Received: 29 August 2019

Accepted: 31 December 2019

Published: 07 January 2020

Copyright (c) 2020 by the author(s). Licensee Hapres, London, United Kingdom. This is an open access article distributed under the terms and conditions of Creative Commons Attribution 4.0 International License.

\begin{abstract}
Compared to mice with the same body mass, naked mole-rats have a lower metabolic rate, higher homeostasis-maintaining activities, higher oxidative damage levels, but a longer lifespan. These observations have raised serious challenges to the widely accepted oxidative stress theory of aging, which suggests a negative correlation between damage levels and lifespan. Here, we introduce a simple theoretical model based on energy conservation and the oxidative stress theory. Employing the model and the physiological parameters of mice and naked mole-rats, we explain why naked mole-rats have higher damage levels despite their higher somatic maintenance efforts; why damage levels in naked mole-rats seem not to change over age; and how these factors concertedly result in a longer lifespan in naked mole-rats. Our results highlight the energy tradeoff between biosynthesis and somatic maintenance, and suggest that the rate of damage accumulation over age and the existence of a threshold of damage for death are the keys to solve the paradox raised by naked molerats.
\end{abstract}

KEYWORDS: energy tradeoff; biosynthesis; proteostasis; comparative study

\section{INTRODUCTION}

The oxidative stress theory, the most widely accepted proximate theory of aging, suggests that the damage to macromolecules caused by deleterious productions of oxidative metabolism (e.g., Reactive Oxygen Species, ROS) is associated with the process of aging [1-3]. Thus, the theory proposes a negative correlation between the oxidative damage level and the lifespan of organisms. The theory has received strong supports as well as serious challenges (see reviews in [4] and [5]). While many empirical 
challenges have been reconciled with the theory by quantitative analyses and qualitative clarifications [4,5], the puzzle raised by the comparative studies on naked mole-rats (Heterocephalus glaber) and CB6F1 mice still remain unsolved [6-10]. Naked mole-rats (NMR, hereafter) have a maximum lifespan of $\sim 32$ years [6,7], 8-10-fold of that of mice with the same body weight. A series of recent empirical works [9-11] have found two lines of evidence from NMR that seemingly disagree with the oxidative stress theory.

First, the antioxidant defense mechanism in NMR is not superior compared to the physiologically age-matched CB6F1 mice [11]. While some branches of the mechanism (such as $\mathrm{Cu} / \mathrm{Zn}$ superoxide dismutase (SOD), Mn SOD, and catalase) are generally 1.3-2 times higher in NMR than those in mice, which nonetheless cannot explain the 8-10-fold difference in lifespan, another branch, cellular glutathione peroxidase, is 70 times lower in NMR. Second, the net oxidative damage level in multiple organs are consistently higher in NMR than in the age-matched mice throughout a large portion of the lifespan of both species, including 2-fold greater lipid peroxidation (isoprostanes) and damage (malondialdehyde) [9], 2-8-fold greater DNA damage $(8-\mathrm{OHdG})$, and 1.5-2-fold greater protein carbonyls [10].

The complex correlations between antioxidant strength and lifespan have been seen previously. Many studies have found positive [12], negative [13-15], or no correlations [15,16] between the antioxidant strength and lifespan in multiple animal models. As pointed out previously [4,5], the disparity between the antioxidant defenses and lifespan does not present a serious challenge to the oxidative stress theory, because the theory does not make a direct connection between these two. What the theory associates the aging process with is the net oxidative damage. The net damage is an integrative and collective end result of the ROS producing, ROS scavenging (including all the antioxidant defense lines), and damage repair (including the molecule chaperons and proteolytic machinery, the enzymes that repair protein and DNA damage) [5]. Thus, the level of antioxidant effort may not correlate with the net damage level directly, and therefore cannot be used as an indicator for lifespan.

However, the challenge to the theory from the second line is more fatal, in which the net damage to various macromolecules were directly assayed and found to be higher in NMR than in age-matched mice. Some efforts have been made to explain how NMR cope with the high oxidative damage and live a long life. Studies have found [9,17-19] that fibroblasts from NMR are more resistant to cytotoxins (such as paraquat, a strong oxidant) than those from mice are, probably through the pathways of p53 and nuclear factor erythroid 2-related factor-2 (Nrf2); NMR have enhanced chaperone activities that repair the misfolded and unfolded proteins as well as assist proteolytic degradation; old NMR are able to keep the proteasomal activities at higher level compared to age-matched old mice; and the levels of lipid and protein damage change negligibly with age. It was also 
proposed [6] that ROS may have beneficial effect in NMR through the hormetic mechanisms that keep cellular homeostasis, enhance macrophage activity, and keep low body temperature, all contributing to longevity.

Among these hypotheses and empirical findings, only the finding that damage level in NMR insignificantly increases over age partially agrees with the oxidative stress theory. The others help to understand how NMR endure the oxidative stress, but do not answer the question raised by the oxidative stress theory: how does high molecular damage lead to a longer lifespan in NMR? Besides NMR, the animals with genetic knock-down manipulation also showed no correlation between oxidative damage and lifespan [20,21]. For example, the mice with a major antioxidant scavenger knocked out (SOD2 ${ }^{+-}$) have the same lifespan as the wild type, but have a higher damage level [21]. Considering these paradox, the leading researchers in the field have asked if the oxidative stress theory is dead [20], and suggested that the results from the comparative studies on oxidative damage and lifespan "challenge the validity of this theory" [8,22], "defy the theory" [6], and "perhaps, it is not oxidative stress that modulates health-span and longevity, but other cytoprotective mechanisms that allow animals to deal with high levels of oxidative damage" [7].

Employing a simple quantitative model based on the first principles of energy conservation and within the framework of the oxidative stress theory, here we use the physiological data from CB6F1 mice and NMR to propose an explanation on how NMR keep higher damage level, and maintain the increase of damage as age at a slower rate, compared to mice. We further show that the correlation between lifespan and oxidative damage measured at certain ages is not always negative, and a positive or no correlation does not necessarily defy the oxidative stress theory of aging. As Rodriguez, Wywial [8] pointed out, "most comparative studies compare data from young healthy adults providing a snap shot of oxidative damage at a given age of each species.” Our model suggests, and numerous empirical data support, that lifespan of an organism largely depends on the trend (rate) of damage increasing throughout life.

\section{THE THEORETICAL MODEL}

The development and verification of the model have been published [5,23-26]. Here we give a brief description of the key assumptions and results of the model. The detailed derivation of the model and a table of the variables and the parameters are available in the Supplementary file.

The model has three assumptions. First, the rate of raw damage production, $H$, is proportional to the resting metabolic rate, $B_{\text {rest }}$ (equivalent to oxygen consumption rate at rest), as $H=\delta \times B_{\text {rest }}$, where $H$ is in unit of mole or gram of damaged biomass per unit time, $B_{\text {rest }}$ is in unit of energy per unit time at rest, and $\delta$ is a constant within a species. The proportionality between $H$ and $B_{\text {rest }}$ comes from the following relationships. The raw damage production rate $H$ is proportional to the 
total metabolic rate, $B_{\text {tot }}$ (total oxygen consumption), which includes the energy expenditure at rest and the energy spent on activities. Numerous empirical data have shown that at least for birds and mammals, the total metabolic rate is proportional to the resting metabolic rate with a constant $f$ [27-30], i.e., $B_{\text {tot }}=f \times B_{\text {rest. }}$ The daily average of $f$ is around 2-3, and is roughly a constant with in taxon [27-30]. Thus, although $H$ estimates the total raw damage production that is associated with the total metabolic rate, it can be expressed in terms of resting metabolic rate, using the proportionality $B_{\text {tot }}=f \times B_{\text {rest }}$. This way the activities' contribution to damage is included in $H=\delta \times B_{\text {rest }}$. Two factors contribute to $\delta$ : the amount of ROS produced as one unit of ATP is produced, which varies slightly due to mitochondrial uncoupling [31]; and more importantly, the amount of cellular damage caused by one unit of un-scavenged ROS. In the following section, we will use protein damage as an example to show that facing the same amount of ROS, different protein qualities may lead to different levels of damage, and therefore different values of $\delta$.

Second, organisms have mechanisms to repair the damage, which cost energy. The amount of damage that can be repaired is proportional to one unit of metabolic energy allocated to repair as $R=\eta B_{\text {rep }}$, where $R$ is the amount of damaged mass that is repaired, $B_{\text {rep }}$ is the metabolic energy allocated to repairing, and $\eta$, a constant within a species, reflects how efficient the repairing mechanism is. We will taking protein stability as an example to show that $\eta$ may vary across species.

It is important to notice that the resting metabolic energy, $B_{\text {rest }}$, is generally partitioned between the energy allocated to repair (somatic maintenance), $B_{\text {rep }}$, and the energy to synthesize new biomass, $B_{\text {syn }}$ including growth and reproduction, i.e., $B_{\text {rest }}=B_{\text {rep }}+B_{\text {syn }}[32,33]$. Here we introduce a parameter that plays a key role in the following analysis: the energy required to synthesize one unit of biomass, $E_{\mathrm{m}}$, which quantifies how expensive the biosynthesis is, i.e., $B_{\text {syn }}=E_{\mathrm{m}} \times G$, where $G$ is growth rate in the unit of accumulated biomass over a unit of time. For the same amount of energy allocated to biosynthesis ( $\left.B_{\mathrm{syn}}\right)$, a large $E_{\mathrm{m}}$ would lead to a slow growth rate $G$. Considering this energy partition, the repair effort can be rewritten as $R=\eta B_{\text {rep }}=\eta\left(B_{\text {rest }}-E_{\mathrm{m}} G\right)$.

The rate of net damage production, $H-R$, therefore, is $\delta B_{\text {rest }}-\eta\left(B_{\text {rest }}-E_{\mathrm{m}} G\right)=(\delta-\eta) B_{\text {rest }}+\eta E_{\mathrm{m}} G$. Damage accumulates over time. Thus, the total net damage at a certain age, $t$, can be estimated as the integral of the rate over time. Approximately the result of the integration is $(\delta-\eta) B_{\text {rest }} \times t+\eta E_{\mathrm{m}}\left(m(t)-m_{0}\right)$ [25], where $m_{0}$ is the body mass at birth $(t=0)$. This equation estimates the damage accumulated over the whole lifetime, including both growth and adult stages. It indicates that the damage at age $t$ is contributed by two terms. The first one is caused by total metabolic energy consumed by organisms from birth to age $t, B_{\text {rest }} \times t$. The second term comes from the energy spent on biosynthesis, $E_{\mathrm{m}} \times\left(m(t)-m_{0}\right)$. Here, $m(t)-m_{0}$ is the total biomass accumulated from birth to age $t$ (total 
growth). Multiplying by the energy required to synthesize one unit of biomass $E_{\mathrm{m}}$, this term gives the total energy allocated to biosynthesis from birth to age $t$. It is intuitive to see that this term's contribution to damage is positive: the total metabolic energy is conserved, and is partitioned between energy for repair and energy for biosynthesis. Thus, more energy spent on biosynthesis means less energy allocated to repair, which in turn results in higher damage level.

To compare different species or individuals within a species, we need to estimate the mass-specific damage, denoted as $D(t)$. Dividing the integral above by the body mass, $m(t)$, it is straightforward to have $D(t)$, the massspecific damage as a function of age $t$,

$$
D(t)=(\delta-\eta) B_{\mathrm{ms}} t+\eta E_{\mathrm{m}}\left(1-m_{0} / m(t)\right)
$$

where $B_{\mathrm{ms}}$ is the mass-specific resting metabolic rate, $B_{\mathrm{ms}}=B_{\text {rest }} / m(t)$, and the term $1-m_{0} / m(t)$ comes from $\left(m(t)-m_{0}\right) / m(t)$.

Third, Equation 1 calculates the mass-specific damage level as a function of age. Here we need an extra assumption to employ Equation 1 for estimating lifespan: animals die when the damage level reaches a threshold, which is a constant within a taxon. i.e., $D(L S)=C$, where $L S$ is the lifespan and $C$ is the threshold of the damage level. Noticing the body mass at $t=L S$ is usually the adult mass $M$, Equation 1 gives

$$
(\delta-\eta) B_{\mathrm{ms}} L S+\eta E_{\mathrm{m}}\left(1-m_{0} / M\right)=C
$$

The threshold of damage that is required for manifest losses in function of organs was also proposed by [34]. Although the value of the damage threshold $C$ is not available, Equations 1 and 2 still make a series of quantitative predictions for comparative studies, as long as the value of $C$ is the same for the animals that are of comparison. In previous studies, we have shown that Equations 1 and 2 correctly predicted more than $80 \%$ of the variations of lifespan across species [5], across breeds within one species [35], and between diet restricted and ad libitum mice and rats [25] (The $80 \%$ of variation refers to the $R^{2}$ values of the data fitting with our model, which contains only one free parameter $-\eta / \delta$ ) The predictions are strongly and quantitatively supported by data collected from several hundreds of empirical studies. Here we show that the same model with the unique parameters of naked mole-rat (NMR) offers simple qualitative answers to the puzzle raised by the comparative studies on mice and NMR.

\section{THE HIGH ENERGETIC COST OF GROWTH IN NAKED MOLE RAT (NMR)}

NMR and mice have similar adult body mass, 30 grams, but it takes 10 months to one year for NMR to fully reach the adult mass [36], whereas mice reach the adult mass in 3-4 months. The large difference in growth rate cannot be fully attributed to the difference in their metabolic rates. The energy for biosynthesis comes from the resting metabolic energy. As mentioned above, different energetic growth models suggested [32,33,37] 
that the resting metabolic energy can be partitioned between the energy allocated to maintenance and that allocated to biosynthesis, i.e., $B_{\text {rest }}=B_{\text {rep }}$ $+E_{\mathrm{m}} G$. Thus, the level of $B_{\text {rest }}$ affects the growth rate $G$. The data on metabolic rate over the full ontogeny of NMR is not available. It is commonly assumed that resting metabolic rate scales with body mass to a $3 / 4$ power, $B_{\text {rest }}=B_{0} m(t)^{0.75}$, as seen in numerous mammalian species [32,38,39], where $B_{0}$ is the normalization coefficient (in unit of energy/time/mass ${ }^{0.75}$ ), and $m(t)$ is body mass over ontogeny. Using the adult resting metabolic rate and the $3 / 4$ scaling power, $B_{\text {rest }}$ (adult) $=B_{0} M^{3 / 4}$, where $M$ is the adult mass, it can be estimated that for NMR, $B_{0}=1230$ Joules/day/gram ${ }^{0.75}$ [40]. The studies on mice metabolic rate gives, on average, $B_{0, \text { mice }}=1550$ Joules $/$ day $/$ gram $^{0.75}[41,42]$. Thus, for NMR and mice with the same body mass the ratio of their metabolic rate is 0.79 $(=1230 / 1550)$. Obviously this ratio of metabolic rate cannot explain the $\sim 3-$ fold difference in growth rate.

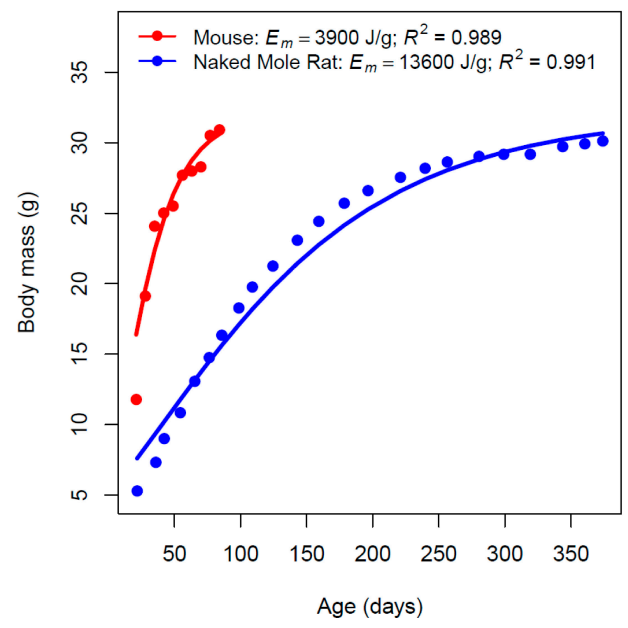

Figure 1. The growth curves of CB6F1 mouse (red) and NMR (blue). Growth data of mouse and NMR are from ENVIGO (http://www.envigo.com, sample size is unkown) and [36] (sample size 80), respectively. The solid curves are the fittings of the growth equation proposed by [32], $m(t)=M \times\left[1-\left(1-\left(m_{0} / M\right)^{0.25}\right) e^{-\left(B_{0} * t\right) /\left(4 E_{\mathrm{m}} M^{0.25}\right)}\right]^{4}$, where birth mass $m_{0}$ is 5 grams for both species, adult $M$ is 32 grams for both species, and the values of $B_{0}$ are given in the text. $E_{\mathrm{m}}$ is the only free parameter, and is obtained by fitting the equation with the growth curves (The details of estimating $E_{\mathrm{m}}$ are available in the Supplementary file).

The rate of the metabolic energy allocated to biosynthesis is the product of the energy required to synthesize and one unit of biomass, and growth rate, i.e., $B_{\mathrm{syn}}=E_{\mathrm{m}} G$. A larger $E_{\mathrm{m}}$ indicates a more expensive growth, i.e., with the same amount energy allocated to growth $\left(B_{\text {syn }}\right)$, a species with a larger $E_{\mathrm{m}}$ would have a slower growth rate. Previous ontogenetic growth models [32,43] proposed a method to estimate the value of $E_{\mathrm{m}}$, using the resting metabolic rate and growth curve of the organisms. Figure 1 shows the fittings of growth curves of NMR and CB6F1 mice with the ontogenetic model (see details in the Supplementary file). The data fitting gives that the values of $E_{\mathrm{m}}$ are 13,600 Joules/gram and 3900 Joules/gram for NMR and 
mice, respectively, meaning to synthesize one unit of biomass, NMR need spend 3.5 times more energy than mice $(13,600 / 3900)$.

Note: Equation $4 \mathrm{~A}$ in the Supplementary file shows how $E_{\mathrm{m}}$ can be estimated using the growth curve and the normalization coefficient of resting metabolic rate $\left(B_{0}\right)$. It shows that $E_{\mathrm{m}}$ changes linearly with $B_{0}$. Empirical data and theoretical considerations [32] show that for many species the resting metabolic rate over ontogeny scales with the body mass to a $3 / 4$ power, i.e., $B_{\text {rest }}=B_{0} m(t)^{3 / 4}$. We assume that it is also true for NMR. Using the adult metabolic rate of NMR and the $3 / 4$ scaling law over ontogeny, we estimate the value of $B_{0}$ by solving this equation: $B_{\text {rest at adult }}=B_{0} M^{3 / 4}$, where $M$ is the adult mass. We then used this value of $B_{0}$ and the growth curve of NMR to estimate $E_{\mathrm{m}}$. However, over ontogeny, the metabolic scaling power may not be exactly $3 / 4$. If the scaling power is larger (smaller) than $3 / 4$, the value of $B_{0}$ would be smaller (larger) than our estimate, and the value of $E_{\mathrm{m}}$, which changes linearly with $B_{0}$, would also be smaller or (larger) than the estimated. In fact, for small rodents, the scaling power is likely to be smaller than 3/4 (e.g., [44]). If it is also the case for NMR, then the value of $E_{\mathrm{m}}$ of NMR would even be higher.

The $E_{\mathrm{m}}$ value of CB6F1 mice is similar to other mammals, such as other strains of mice, dogs and rats [43], whereas the value of NMR is exceptionally high. Historically, the value of $E_{\mathrm{m}}$ was of interest in agriculture [33], fishery [45], and physiological ecology [46], because it indicates the energetic efficiency that animals deposit biomass. Here, and in a few recent publications of ours [5,24], we call upon the biomedical community, especially the biology of aging community, to pay more attention to this trait of animals. We have recently proposed a "cost-quality" hypothesis, suggesting that more expensive biosynthesis results in high quality macromolecules that are more resistant to oxidative insults [47]. The hypothesis is general, and applies to all the macromolecules, including DNA, protein, and lipid. However, compared to the other macromolecules, the energetics of protein synthesis, folding/unfolding/refolding, aggregation, and degradation have been more intensively studied, so here we take protein synthesis and homeostasis (proteostasis) as an example to explain this hypothesis, and apply it to the comparison between NMR and mice.

\section{THE RELATIONSHIP BETWEEN ENERGETIC COST OF GROWTH AND PROTEOSTASIS}

We need to emphasize that conceptually and numerically $E_{\mathrm{m}}$ is different than the energy content of biomass. The latter is the energy deposited in tissues, e.g., 4.1 Cal per gram of protein and 9.5 Cal per gram of lipid, etc., which is measured with calorimetric techniques. $E_{\mathrm{m}}$, however, is the energy required to assemble the monomers to polymers, fold them, transport them to the required location, etc. [30,48]. This energy does not deposit in the tissue. Instead, it dissipates as heat, because it is a fraction of respiration [30]. The variation in $E_{\mathrm{m}}$ across species may not necessarily 
come from the difference in their body composition, because the energy required to deposit a unit of protein is similar to that of fat $[45,48]$, and species with the same body composition can have 20 -fold difference in the values of $E_{\mathrm{m}}$ [47].

We hypothesize that the variations in $E_{\mathrm{m}}$ across species come from the differences in the effort that species makes to synthesize proteins, and a high $E_{\mathrm{m}}$ results in better protein stability. There are multiple mechanisms that connect the energy cost of protein synthesis to protein homeostasis (proteostasis). For example, different amino acid compositions lead to different protein stabilities [49,50], and synthesizing different amino acid requires different amounts of energy [51-53]; Different proofreading efforts also have different energy requirements [54,55], and are tightly associated with protein fidelity [56]. Perhaps more importantly, the species with higher value of $E_{\mathrm{m}}$ may have a lower tolerance to the mistakes, such as misfolding, in the newly synthesized proteins. In those species, such proteins are quickly unfolded and refolded via the chaperon activities, and/or degraded and resynthesized via the proteasomal activities. These activities, especially unfolding and resynthesis, cost considerable amount of the energy, but retard the processes of protein aggregation and directly determine the proteostasis [57-60]. Recalling that $E_{\mathrm{m}}$ reflects how expensive it is to accumulate one unit of biomass, the species, which is "picky" about the newly synthesized protein and unfold/refold and degrade/resynthesize proteins more often than those less "picky" species, would spend more energy to accumulate one unit of biomass (net gain), grow more slowly, but have a slow rate of protein aggregation.

Interestingly, the results from recent studies suggest that naked molerat is such a species, which is facilitated with high level protein damage repair mechanisms. Compared to mice, NMR have a high level of protein involved in the ubiquitin proteasome system, high chaperon activities, and high levels of proteasome activity [7,8,17,19,22]. These traits, which maintain at high levels throughout NMR's life $[8,17]$, on one hand makes growth more energetically costly during development, and on the other hand greatly contribute to NMR's proteostasis.

Nonetheless, the results of these studies still cannot answer three questions: (1) why do NMR have higher oxidative damage level than mice despite these proteostasis-maintaining machinery, (2) why do the damage levels in NMR seem not change over age, and (3) how do those factors concertedly result in a longer lifespan in NMR. In the next section, we use our model (Equations 1 and 2) to offer simple yet novel answers to these questions.

\section{SLOW PROGRESS OF DAMAGE LEVEL LEADS TO LONGER LIFESPAN}

Two parameters in our model are possibly affected by $E_{\mathrm{m}}$, namely, the amount of net damage caused by one unit of metabolic energy produced 
$(\delta)$ and the amount of damage that can be repaired by one unit of energy allocated to repair $(\eta)$.

It is well known that proteins' folding property greatly affects their susceptibility to oxidative damage, probably because tightly packed side chains in the well-folded state provides better protection from ROS and other insults [61-65]. Thus, facing the same level of ROS (presumably associated with similar amount of ATP produced), the species with a higher $E_{\mathrm{m}}$ produces less amount of damage, and has a lower value of $\delta$, because it is less vulnerable to the oxidative insults.

The relationship between $E_{\mathrm{m}}$ and $\eta$ is less straightforward. From the viewpoint of evolutionary optimization, in a species with high $E_{\mathrm{m}}$, selection would promote the machinery that repair more damage with one unit of energy (higher $\eta$ ), i.e., a low value of $\eta$ would mismatch the high $E_{\mathrm{m}}$ economically. Proximately, different branches of the proteostasis maintenance mechanism have different efficiencies [57,65]. A species equipped with high levels of chaperon and proteasomal machinery, such as NMR, is presumably able to more efficiently prevent protein unfolding and aggregation, and also degrade more aggregated proteins with a unit of energy allocated to maintenance (higher $\eta$ ).

We need to emphasize on three points about $\delta$ and $\eta$. First, the absolute values of these two parameters are not available. But from fitting the data on longevity and oxidative damage as functions of ontogenetic body mass and metabolic rate, it has been estimated that the ratio of them, $\eta / \delta$, is smaller but very close to 1.0. Species with low $E_{\mathrm{m}}$, such as hawkmoth larvae (hornworms) with $E_{\mathrm{m}} \approx 300$ Joules/gram, has a ratio $\eta / \delta \approx 0.96$ [23], whereas species with high $E_{\mathrm{m}}$, such as small rodents and dogs with $E_{\mathrm{m}} \approx$ 5000 Joules/gram, have the ratio $\eta / \delta \approx 0.99$ [25,35]. Second, the quantitative relationship between $E_{\mathrm{m}}$ and $\delta$ and $\eta$ are not known. The analysis we gave above, as well as the results from previous studies [23,25,35], give a qualitative suggestion that as $E_{\mathrm{m}}$ increases, the value of $\delta$ decreases and that of $\eta$ increases. But because damage accumulates, $\delta$ (raw damage) should be always larger than $\eta$ (repair), otherwise the net damage level may decrease with age. Third, the changes in $\delta$ and $\eta$ due the change in $E_{\mathrm{m}}$ are significant but small. Organisms have efficient damage repair mechanisms, and even species such as hornworm, which has a very low $E_{\mathrm{m}}$, still has a relatively high ratio of $\delta / \eta$. We qualitatively describe these trends with $E_{\mathrm{m}}$ in Figure 2. 


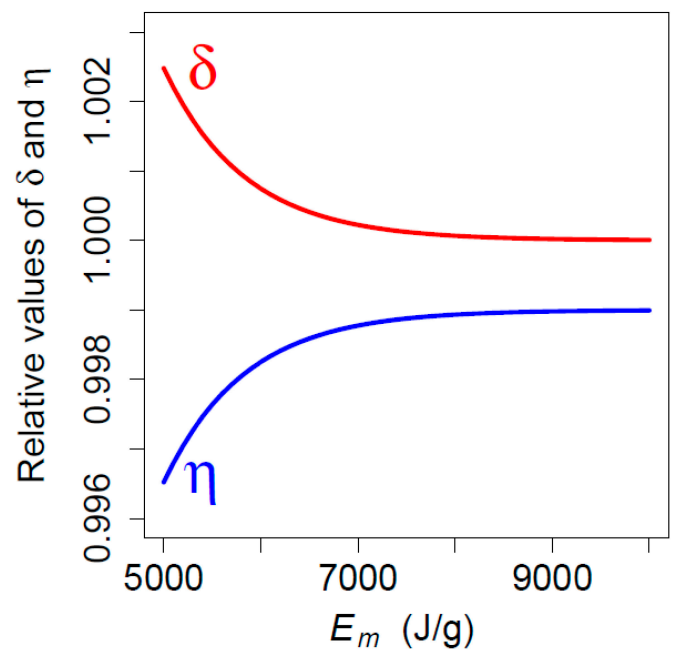

Figure 2. A qualitative description of how the values of $\boldsymbol{\delta}$ and $\boldsymbol{\eta}$ change as $\boldsymbol{E}_{\mathrm{m}}$. The curves are produced by the assumptions that $\delta=1+e^{-0.0012 E_{\mathrm{m}}}$ and $\eta=0.999-e^{-0.0012 E_{\mathrm{m}}}$. The assumptions capture the three features of $\delta$ and $\eta$, i.e., the ratio of them is smaller and close to 1.0; $\delta$ decreases and $\eta$ increases with $E_{\mathrm{m}}$; and the decrease and the increase in them with $E_{\mathrm{m}}$ are slow.

Overall, compared to mice, NMR have a 3.5-time higher $E_{\mathrm{m}}$, a 0.79-time lower mass-specific metabolic rate $\left(B_{\mathrm{ms}}\right)$, a lower $\delta$, a higher $\eta$, and therefore a lower $(\delta-\eta)$. Putting all these parameters into Equation 1, $D(t)=(\delta-\eta) B_{\mathrm{ms}} t+\eta E_{\mathrm{m}}\left(1-m_{0} / M\right)$, we can compare the damage levels of mice and NMR as functions of age during the adulthood, as shown in Figure 3. Note, during adulthood, $m(t)$ in Equation 1 becomes adult mass $M$, and $m_{0} / M$ is the birth and adult mass ratio, which is roughly the same in NMR and mice.

Equation 1 indicates that two terms contribute to the damage. The second term, $\eta E_{\mathrm{m}}\left(1-m_{0} / M\right)$, estimates the amount of damage accumulated during growth, and gives the height of the damage level at the beginning of the adulthood. Mice and NMR have the same birth mass $\left(m_{0}\right)$ and the same adult mass $(M)$, but NMR have a higher $\eta E_{\mathrm{m}}$. Thus, NMR have a higher level of damage at the beginning of the adulthood (the blue curve in Figure $3 \mathrm{~A})$. As we explained earlier, intuitively if more energy is allocated to growth, less will be available for damage repair, and the damage accumulated during growth will be higher. The first term in Equation 1 is a function of age $t$, and the slope is $(\delta-\eta) B_{\mathrm{ms}}$. Compared to mice, NMR have a smaller $(\delta-\eta)$ and a smaller $B_{\mathrm{ms}}$, and therefore a much shallower slope (the blue curve in Figure 3A), so that the damage levels seem to be almost unchanged over age $[9,10,17]$.

Thus, our theoretical model offers simple and clear physiological explanations to the empirical observations. The higher level of damage in NMR comes from its higher $\eta E_{\mathrm{m}}$, which reflects the tradeoff between the efforts of growth and damage repair during development. The difference in the slopes, $(\delta-\eta) B_{\mathrm{ms}}$, between NMR and mice explains why the damage in mice increases relatively fast in a linear pattern as age [9], whereas the changes in lipid and protein damage level in NMR are hardly detectable as 
age $[9,10,17]$. Moreover, since mice have a low damage level at the beginning of the adulthood and a steeper slope, and the opposite in NMR, the damage curves of these two species will cross at a certain age (the black dot in Figure 3A). Our model suggests that this crossing point happens later in the mice's life. The assays on both species were probably conducted before the crossing, and therefore failed to show it (the same level of damage in both species in Figure 3A).

More importantly, our model predicts that if the crossing point is below the death threshold (the horizontal dash line in Figure 3B), the damage level of mice will reach the threshold earlier than that of NMR, and results in a shorter lifespan. Figure 3B also suggests that there exists a short time window between the crossing point and the damage threshold, in which mice has a higher damage level than NMR. We need to emphasize that Figure 3 is qualitative, because the exact values of $\delta$ and $\eta$ are unknown. Nonetheless, it offers physiological and first-principle explanations to the observed empirical patterns of damage in NMR and mice, and meanwhile answers the question raised by the oxidative stress theory: why does NMR have higher level of damage but a longer lifespan than mice.
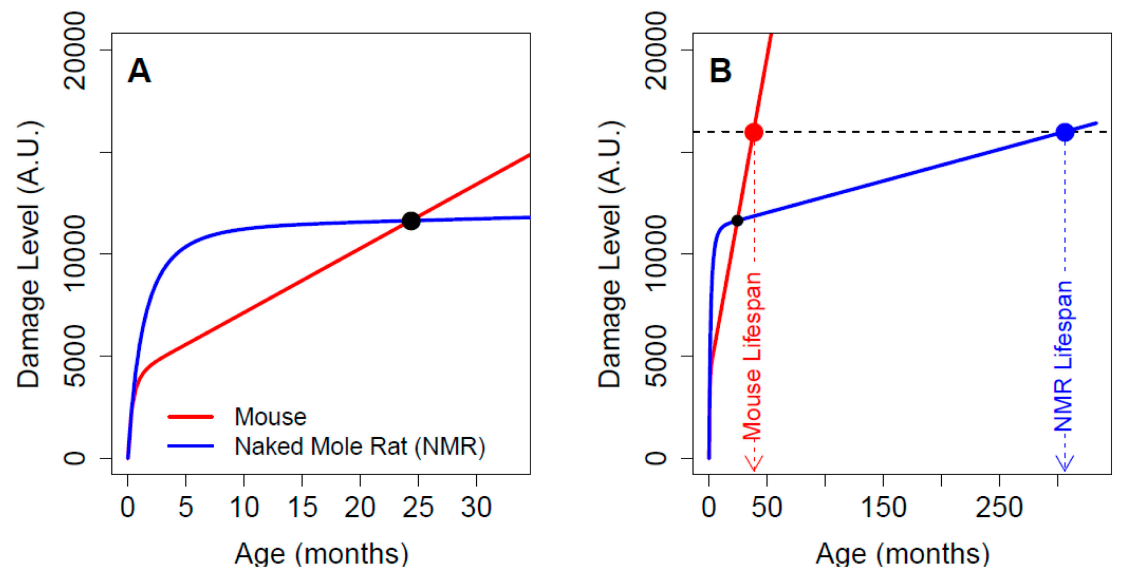

Figure 3. The estimated damage levels of mice and NMR by Equation 1. (A) The damage levels in young adults. (B) The damage levels throughout the lives of mice and NMR. The horizontal dash line is the damage threshold of death. Note, the values of damage in this figure are relative and are in arbitrary unit (A.U.). We used the following values of the parameters in Equation 1 to plot the damage curves: $B_{\mathrm{ms}}=B_{0} m(t)^{-1 / 4}$, where the values of $B_{0}$ for NMR and mouse are 1230 and 1550 Joules/day/gram ${ }^{0.75}$, respectively. The body mass curve, $m(t)$, is the same as in Figure 1; the birth mass $\left(m_{0}\right)$ of NMR and mouse is grams, and the adult mass $(M)$ of both species is 32 grams; the values of $E_{\mathrm{m}}$ of NMR and mouse are 13,600 and 3900 Joules/gram, respectively; and the values of $\delta$ and $\eta$ are the same as in Figure 2.

A clarification needs to be made here. We hypothesized that in the species with a high $E_{\mathrm{m}}$, the macromolecules of superior resistance to damage are synthesized or incorrectly synthesized molecules are extensively resynthesized. At the first glance, this seems to be at odds with the high rate of damage measured early on in the adulthood. Here is the explanation. A higher $E_{\mathrm{m}}$ is associated with a slightly lower $\delta$ and a slightly higher $\eta$, as suggested in Figure 2. Both $\delta$ and $\eta$ are related to one unit of 
energy, i.e., for one unit of energy produced, the associated raw damage is lower (due to the lower $\delta$ ), and for one unit of energy allocated to maintenance, the amount of damaged macromolecules that can be repaired or degraded is higher (due to the higher $\eta$ ). However, a three-time higher $E_{\mathrm{m}}$ (NMR/mouse) also means that, mass-specifically, the energy allocated to growth (biosynthesis) is three times higher in NMR. Recall that resting metabolic rate is partitioned between the rates of energy allocated to biosynthesis and that to somatic maintenance, $B_{\text {rest }}=B_{\text {syn }}+B_{\text {rep }}$. Since NMR have a lower resting metabolic rate ( $\left.B_{\text {rest }}\right)$ and three-time higher $E_{\mathrm{m}}$ than mice, the energy available for maintenance $\left(B_{\text {rep }}\right)$ during growth is greatly reduced in NMR, compared to mice. Thus, although for one unit of energy allocated to maintenance, NMR can repair/degrade slightly more damage (a higher $\eta$ ), it has much less energy allocated to maintenance during growth compared to mice. Consequently during growth, NMR accumulates higher damage.

The damage levels of mice and NMR shown in Figure 3 are plotted as functions of chronological age, whereas the empirical studies compared the damage levels of these species at the matched physiological ages $[9,10]$. In one of these studies, the matched ages for mice and NMR are: 4-mo vs 30 -mo; 12 -mo vs $75-\mathrm{mo} ; 18$-mo vs $130-\mathrm{mo}$, and 24 -mo vs 180 -mo [9]. The ratio of the chronological ages is roughly 7 . The matched physiological age is equal to the chronological age of each species divided by the maximum lifespan of each species. To compare our model's prediction and the empirical results, in Figure 4 we replot the same damage curves from Figure 3B at the relative age scales, i.e., the age of NMR (the blue horizontal axis on the top) is scaled down by roughly seven times to match the age of mice (the red horizontal axis at the bottom), so that their maximum lifespans appear at the same relative time in the figure. The blue and red dots in the figure are the predicted damage at their matched-ages (4-, 12-, 18-, 24-mo for mice, and 30-, 75-, 130-, 180-mo for NMR). Clearly, at each matched-age our model predicts that damage in NMR is higher than that in mice, agreeing with the empirical data $[9,10]$. Interestingly, our model also predicts that difference in the damage between these two species gets smaller as age. The assays on old and senescent adult mice and NMR, e.g., the urinary isoprostane level and malondialdehyde level, did suggested a vanishing difference as age [9]. 


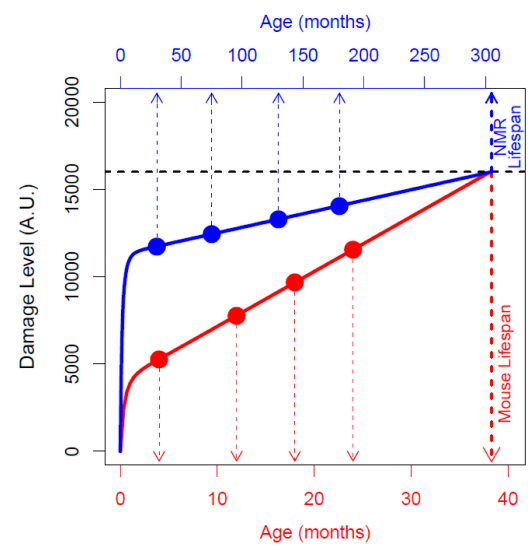

Figure 4. Physiological age-matched damage levels of mice and NMR. Since the age scales are matched, the two species reach the lifespan at the same point where the threshold is reached. Because of the different time scale, the chronological crossing point in Figure 3 does not show in this figure.

\section{FUTURE DIRECTION}

We have shown that the combination of the crossing of the damage curves and the assumption of the damage threshold offers a simple answer to the question why NMR have higher oxidative damage but live longer than mice. Previously we have applied the same idea to explain another paradox raised by the gene knock-out studies [5]. Manganese-dependent superoxide dismutase (MnSOD, or SOD2) is an important antioxidant enzyme. It has been shown [21] that $S O D 2^{+/-}$knocked out mice have higher DNA damage than the wild type, but the same lifespan. We noticed that, although the damage in $\mathrm{SOD2}^{+/-}$is higher in multiple organs, the increase of the damage with age is slower in the knockout mice than that of the wild type [21], a similar pattern that we saw in the comparison between mice and NMR. We postulated [5] that the damage curves of the knockout and the wild type probably cross around the damage threshold of death, so that they reached the lifespan at the same time.

We believe the model and analysis presented in this study open a door to various future researches. Here we suggest four lines of future studies to further test the model. First, we estimated the value of $E_{\mathrm{m}}$ of the naked mole-rat based on the empirical growth curve and metabolic rates measured at four different body masses that are very close to the adult mass [40]. For a more accurate estimate, measurements of metabolic rate throughout the development with higher temporal resolution are required.

Second, although one study [9] arguably showed a dismissing difference in damage between mice and NMR, which supports our prediction in Figure 4, it also showed that lipid peroxide level had no increase from intermediate to old age in NMR, which is seemingly against the slow progress of damage that is predicted by our model. However, this empirical result only came from one marker (MDA) in one organ (liver) at two time points (intermediate and old). It is possible that the damage in NMR increases so slowly that the low time-resolution data on one oxidative damage marker from one organ may not detect the small 
increase. We therefore call for future oxidative damage assays on more markers with higher temporal resolution are need to confirm this pattern. With more data points throughout the whole life, we expect to see a slight increase of damage level in NMR, and the difference in damage levels between mice and NMR at matched physiological ages will get smaller as shown in Figure 4.

Third, it is probably difficult, if possible, to change the damage threshold for death, because it is the collective result of multiple physiological processes at cellular and organismal level. But, it should be easier to experimentally manipulate the damage curves of both specie, so that the crossing point gets closer to or farther away from the damage threshold. For example, if one can experimentally raise the damage levels of both species by the same percentage, so that the crossing point gets closer to, or even above the damage threshold, then the difference in their lifespan will be smaller, and the reduction in lifespan of NMR will be more severe than that of mice.

Finally, our theoretical model is not species-specific, and the predictions have been supported by numerous data from mammals and insects [5,23-25,35]. Thus, we hypothesize that the pattern of damage and its effect on lifespan as shown in Figures 3 and 4 would be seen in comparisons of other species, which have similar body masses but significantly different lifespans. One such example would be long-living turtle and short-living lizard [66]. Future comparative studies on such species will provide valuable data to test the universality of the oxidative stress theory in general and our theoretical model specifically.

\section{SUPPLEMENTARY MATERIAL}

The supplementary material is available online at https://doi.org/10.20900/agmr20200006.

\section{AUTHOR CONTRIBUTIONS}

$\mathrm{CH}$ developed the model and conceptualized the study; WZ, XT, and $\mathrm{CH}$ conducted the discussions; WZ and $\mathrm{CH}$ made the simulations; WZ, XT, and $\mathrm{CH}$ wrote the paper.

\section{CONFLICTS OF INTEREST}

The authors declare that there is no conflict of interest.

\section{FUNDING}

X.L. Tang was supported by the National Natural Science Foundation of China (No. 31501860) and Fundamental Research Funds for the Central Universities (lzujbky-2017-150). 


\section{REFERENCES}

1. Sohal RS, Mockett RJ, Orr WC. Mechanisms of aging: an appraisal of the oxidative stress hypothesis. Free Radical Biol Med. 2002;33(5):575-86.

2. Balaban R, Nemoto S, Finkel T. Mitochondria, Oxidants, and Aging. Cell. 2005;120(4):483-95.

3. Hulbert AJ, Pamplona R, Buffenstein R, Buttemer WA. Life and Death: Metabolic Rate, Membrane Composition, and Life Span of Animals. Physiol Rev. 2007;87:1175-213.

4. Barja G. Updating the Mitochondrial Free Radical Theory of Aging: An Integrated View, Key Aspects, and Confounding Concepts. Antioxid Redox Signal. 2013;19(12):1420-45.

5. Hou C, Amunugama K. On the complex relationship between energy expenditure and longevity: Reconciling the contradictory empirical results with a simple theoretical model. Mech Ageing Dev. 2015;149(0):50-64.

6. Saldmann F, Viltard M, Leroy C, Friedlander G. The Naked Mole Rat: A Unique Example of Positive Oxidative Stress. Oxidative Med Cell Longev. 2019;2019:4502819. doi: 10.1155/2019/4502819

7. Lewis KN, Andziak B, Yang T, Buffenstein R. The naked mole-rat response to oxidative stress: just deal with it. Antioxid Redox Signal. 2013;19(12):1388-99.

8. Rodriguez KA, Wywial E, Perez VI, Lambert AJ, Edrey YH, Lewis KN, et al. Walking the oxidative stress tightrope: a perspective from the naked mole-rat, the longest-living rodent. Curr Pharm Design. 2011;17(22):2290-307.

9. Andziak B, Buffenstein R. Disparate patterns of age-related changes in lipid peroxidation in long-lived naked mole-rats and shorter-lived mice. Aging Cell. 2006;5(6):525-32.

10. Andziak B, O'Connor TP, Qi W, DeWaal EM, Pierce A, Chaudhuri AR, et al. High oxidative damage levels in the longest-living rodent, the naked mole-rat. Aging Cell. 2006;5(6):463-71.

11. Andziak B, O'Connor TP, Buffenstein R. Antioxidants do not explain the disparate longevity between mice and the longest-living rodent, the naked mole-rat. Mech Ageing Dev. 2005;126(11):1206-12.

12. Ono $\mathrm{T}$, Okada $\mathrm{S}$. Unique increase of superoxide dismutase level in brains of long living mammals. Exp Gerontol. 1984;19(6):349-54.

13. Perez-Campo R, Lopez-Torres M, Cadenas S, Rojas C, Barja G. The rate of free radical production as a determinant of the rate of aging: evidence from the comparative approach. J Comp Physiol B. 1998;168:149-58.

14. Barja G, Cadenas S, Rojas C, Lopez-Torres M, Perez-Campo R. A decrease of free radical production near critical targets as a cause of maximum longevity in animals. Comp Biochem Physiol B. 1994;108(4):501-12.

15. Page MM, Richardson J, Wiens BE, Tiedtke E, Peters CW, Faure PA, et al. Antioxidant enzyme activities are not broadly correlated with longevity in 14 vertebrate endotherm species. Age. 2010;32(2):255-70.

16. Lopez-Torres M, Perez-Campo R, Rojas C, Cadenas S, Barja G. Maximum life span in vertebrates: relationship with liver antioxidant enzymes, glutathione system, ascorbate, urate, sensitivity to peroxidation, true malondialdehyde, 
in vivo $\mathrm{H}_{2} \mathrm{O}_{2}$, and basal and maximum aerobic capacity. Mech Ageing Dev. 1993;70(3):177-99.

17. Pérez VI, Buffenstein R, Masamsetti V, Leonard S, Salmon AB, Mele J, et al. Protein stability and resistance to oxidative stress are determinants of longevity in the longest-living rodent, the naked mole-rat. Proc Nat Acad Sci U S A. 2009;106(9):3059-64.

18. Salmon AB, Akha AAS, Buffenstein R, Miller RA. Fibroblasts from naked molerats are resistant to multiple forms of cell injury, but sensitive to peroxide, ultraviolet light, and endoplasmic reticulum stress. J Gerontol Ser A. 2008;63(3):232-41.

19. Lewis KN, Mele J, Hornsby PJ, Buffenstein R. Stress resistance in the naked mole-rat: the bare essentials-a mini-review. Gerontology. 2012;58(5):453-62.

20. Pérez VI, Bokov A, Remmen HV, Mele J, Ran Q, Ikeno Y, et al. Is the oxidative stress theory of aging dead? Biochim Biophys Acta. 2009;1790(10):1005-14.

21. Van Remmen H, Ikeno Y, Hamilton M, Pahlavani M, Wolf N, Thorpe SR, et al. Life-long reduction in MnSOD activity results in increased DNA damage and higher incidence of cancer but does not accelerate aging. Physiol Genomics. 2003;16(1):29-37.

22. Kim EB, Fang X, Fushan AA, Huang Z, Lobanov AV, Han L, et al. Genome sequencing reveals insights into physiology and longevity of the naked mole rat. Nature. 2011;479(7372):223.

23. Amunugama K, Jiao L, Olbricht G, Walker C, Huang Y-W, Nam PK, et al. Cellular oxidative damage is more sensitive to biosynthetic rate than to metabolic rate: A test of the theoretical model on hornworms (Manduca sexta larvae). Exp Gerontol. 2016;82:73-80.

24. Hou C. Increasing energetic cost of biosynthesis during growth makes refeeding deleterious. Am Nat. 2014;184:233-47.

25. Hou C. The energy trade-off between growth and longevity. Mech Ageing Dev. 2013;134(9):373-80.

26. Hou C, Bolt K, Bergman A. A general life history theory for effects of caloric restriction on health Maintenance. BMC Syst Biol. 2011;5(1):78-90.

27. Nagy KA. Field metabolic rate and body size. J Exp Biol. 2005;208(9):1621-5.

28. Peterson CC, Nagy KA, Diamond J. Sustained metabolic scope. Proc Natl Acad Sci U S A. 1990;87(6):2324-8.

29. Nagy KA. Field metabolic rate and food requirement scaling in mammals and birds. Ecol Monogr. 1987;57(2):112-28.

30. Hou C, Zuo WY, Moses ME, Woodruff WH, Brown JH, West GB. Energy uptake and allocation during ontogeny. Science. 2008;322:736-9.

31. Brand MD. Uncoupling to survive? The role of mitochondrial inefficiency in ageing. Exp Gerontol. 2000;35(6-7):811-20.

32. West GB, Brown JH, Enquist BJ. A general model for ontogenetic growth. Nature. 2001;413:628-31.

33. Brody S. Bioenergetics and growth. New York (US): Reinhold; 1945.

34. Sohal RS, Forster MJ. Caloric restriction and the aging process: a critique. Free Radical Biol Med. 2014;73:366-82. doi: 10.1016/j.freeradbiomed.2014.05.015 
35. Fan R, Olbricht G, Baker X, Hou C. Birth mass is the key to understand the negative correlation between lifespan and body size in dogs. Aging. 2016; 8(12): 3209-21.

36. O'Riain M, Jarvis J. The dynamics of growth in naked mole-rats: the effects of litter order and changes in social structure. J Zool. 1998;246(1):49-60.

37. Kooijman S. Dynamic energy budget theory. Cambridge (UK): Cambridge University Press; 2010.

38. Savage VM, Gillooly JF, Woodruff WH, West GB, Allen AP, Enquist BJ, et al. The predominance of quarter-power scaling in biology. Funct Ecol. 2004;18:25782.

39. Brown JH, Gillooly JF, Allen AP, Savage VM, West GB. Toward a metabolic theory of ecology. Ecology. 2004;85:1771-89.

40. O'Connor TP, Lee A, Jarvis JUM, Buffenstein R. Prolonged longevity in naked mole-rats: age-related changes in metabolism, body composition and gastrointestinal function. Comp Biochem Physiol A. 2002;133(3):835-42.

41. Selman C, Lumsden S, Bunger L, Hill WG, Speakman JR. Resting metabolic rate and morphology in mice (Mus musculus) selected for high and low food intake. J Exp Biol. 2001;204(4):777-84.

42. Speakman JR. Measuring energy metabolism in the mouse-theoretical, practical, and analytical considerations. Front Physiol. 2013;4:34.

43. Moses ME, Hou C, Woodruff WH, West GB, Nekola JC, Zuo W, et al. Revisiting a Model of Ontogenetic Growth: Estimating Model Parameters from Theory and Data. Am Nat. 2008;171(5):632-45.

44. Derting TL. Metabolism and Food Availability as Regulators of Production in Juvenile Cotton Rats. Ecology. 1989;70:587-95.

45. Lupatsch I, Kissil GW, Sklan D. Comparison of energy and protein efficiency among three fish species gilthead sea bream (Sparus aurata), European sea bass (Dicentrarchus labrax) and white grouper (Epinephelus aeneus): energy expenditure for protein and lipid deposition. Aquaculture. 2003;225(1-4):17589.

46. Ricklefs RE. Energetics of reproduction in birds. In: Paynter RAJ, editor. Avian Energetics. Cambridge (US): Nuttall Ornithol Club; 1974.

47. Ferral N, Gomez N, Holloway K, Neeter H, Fairfield M, Pollman K, et al. The extremely low energy cost of biosynthesis in holometabolous insect larvae. J Insect Physiol. 2020;120:103988.

48. Morowitz HJ. Foundations of Bioenergetics. New York (US): Academic Press; 1978.

49. Ponnuswamy PK, Muthusamy R, Manavalan P. Amino acid composition and thermal stability of proteins. Int J Biol Macromol. 1982;4(3):186-90.

50. Argos P, Rossman MG, Grau UM, Zuber H, Frank G, Tratschin JD. Thermal stability and protein structure. Biochemistry. 1979;18(25):5698-703.

51. Kafri M, Metzl-Raz E, Jona G, Barkai N. The Cost of Protein Production. Cell Rep. 2016;14(1):22-31.

52. Swire J. Selection on Synthesis Cost Affects Interprotein Amino Acid Usage in All Three Domains of Life. J Mol Evol. 2007;64(5):558-71. 
53. Akashi H, Gojobori T. Metabolic efficiency and amino acid composition in the proteomes of Escherichia coli and Bacillus subtilis. Proc Natl Acad Sci U S A. 2002;99(6):3695-700.

54. Jakubowski H. Energy cost of translational proofreading in vivo. The aminoacylation of transfer RNA in Escherichia coli. Ann N Y Acad Sci. 1994 Nov 30;745:4-20.

55. Blomberg C. Thermodynamic aspects on accuracy in the synthesis of biomolecules. Int J Quantum Chem. 1983;23(2):687-707.

56. Cochella L, Green R. Fidelity in protein synthesis. Curr Biol. 2005;15(14):R53640.

57. Santra M, Farrell DW, Dill KA. Bacterial proteostasis balances energy and chaperone utilization efficiently. Proc Natl Acad Sci U S A. 2017;114(13):E265461.

58. Salway KD, Gallagher EJ, Page MM, Stuart JA. Higher levels of heat shock proteins in longer-lived mammals and birds. Mech Ageing dev. 2011;132(67):287-97.

59. Dahl J-U, Gray MJ, Jakob U. Protein quality control under oxidative stress conditions. J Mol Biol. 2015;427(7):1549-63.

60. Reichmann D, Voth W, Jakob U. Maintaining a healthy proteome during oxidative stress. Mol Cell. 2018;69(2):203-13.

61. Dukan S, Farewell A, Ballesteros M, Taddei F, Radman M, Nyström T. Protein oxidation in response to increased transcriptional or translational errors. Proc Natl Acad Sci U S A. 2000;97(11):5746-9.

62. Girod M, Enjalbert Q, Brunet C, Antoine R, Lemoine J, Lukac I, et al. Structural basis of protein oxidation resistance: a lysozyme study. PLoS One. 2014;9(7):e101642.

63. Vidovic A, Supek F, Nikolic A, Krisko A. Signatures of conformational stability and oxidation resistance in proteomes of pathogenic bacteria. Cell Rep. 2014;7(5):1393-400.

64. Ghosh K, de Graff AM, Sawle L, Dill KA. Role of proteome physical chemistry in cell behavior. J Phys Chem B. 2016;120(36):9549-63.

65. Santra M, Dill KA, de Graff AM. How Do Chaperones Protect a Cell's Proteins from Oxidative Damage? Cell Systems. 2018;6(6):743-51. e3.

66. Patnaik B. Ageing in reptiles. Gerontology. 1994;40(2-4):200-20.

How to cite this article:

Zuo W, Tang X, Hou C. Why Naked Mole-Rats Have High Oxidative Damage But Live a Long Life: A Simple Explanation Based on the Oxidative Stress Theory of Aging. Adv Geriatr Med Res. 2020;2(1):e200006. https://doi.org/10.20900/agmr20200006 